\title{
Does bariatric surgery improve ovarian stimulation characteristics, oocyte yield, or embryo quality?
}

\author{
Abraham Tsur, Raoul Orvieto, Jigal Haas, Alon Kedem and Ronit Machtinger
}

\begin{abstract}
Background: Obesity is a major global health concern associated with multiple co-morbidities. Bariatric surgery has been considered a good treatment option in cases of morbid obesity. This preliminary study aims to investigate the effect of bariatric surgery on ovarian stimulation characteristics and IVF treatment cycle outcome.

Methods: A retrospective study that was performed in a tertiary, university-affiliated medical center and included all patients who underwent IVF treatment both before and after bariatric surgery. Data on ovarian stimulation variables of IVF treatment cycle prior and following the bariatric surgery were reviewed and compared.

Results: From January 2005 to June 2014, seven women fulfilled the inclusion criteria. After the operation, BMI was significantly reduced (mean \pm SD) ( $43.1 \pm 3.3$ vs. $29.6 \pm 7.33, p=0.018$ ), as was the number of gonadotropin ampoules required during stimulation ( $69.3 \pm 10.5$ vs. $44.5 \pm 17, p=0.043)$. No between-cycle differences were observed in peak estradiol level, the number of oocytes retrieved, and percentage of mature oocytes.

Conclusions: To the best of our knowledge, this preliminary case series is the first comparison of IVF cycle characteristics prior to and following bariatric surgery. The operation seems to reduce treatment costs without affecting oocyte or embryo quality. Further large studies are required to establish the surgery's effect on IVF outcome among infertile women.
\end{abstract}

Keywords: Bariatric surgery, Morbid obesity, IVF, Female fertility

\section{Background}

Obesity is a major global health concern with increasing prevalence [1]. According to the National Health and Nutrition Examination Survey (NHANES), the prevalence of obesity [body mass index $(\mathrm{BMI})>30 \mathrm{~kg} / \mathrm{m}^{2}$ ] in the United States between 2011 and 2012 was as high as $36.1 \%$ among adult women, with a $8.3 \%$ prevalence of morbid obesity (BMI $\geq 40)$ [2]. Obesity may be associated with various co-morbidities such as diabetes type 2, chronic hypertension, heart disease, sleep apnea, and stroke [3]. Moreover, being obese may reduce woman's fertility, increase risks associated with pregnancy [4], and be detrimental to in vitro fertilization (IVF) outcomes [5-8].

Treatment options include lifestyle modification, exercise, dietary and pharmacological therapy, liposuction, and bariatric surgery. While non-surgical approaches are generally associated with modest weight loss that is poorly

\footnotetext{
*Correspondence: Ronit.Machtinger@sheba.health.gov.il

Infertility and In Vitro Fertilization Unit, Department of Obstetrics and

Gynecology, Chaim Sheba Medical Center (Tel-Hashomer) and Sackler School of Medicine, Tel Aviv University, Tel Aviv, Israel
}

sustained in most patients, bariatric surgery has been associated with significant weight loss, and its beneficial effects seem to be sustained in the long term [9].

Along the many merits of bariatric surgeries, these procedures might be followed by intraoperative, short and long term complications as wound infection, leakage through staples or sutures, abdominal hernia, ulcers, dumping syndrome, and nutritional deficiencies leading to anemia and osteoporosis [10].

Current indications for this operation are $\mathrm{BMI} \geq$ $40 \mathrm{~kg} / \mathrm{m}^{2}$ without co-morbidities or $\mathrm{BMI} \geq 35 \mathrm{~kg} / \mathrm{m}^{2}$ with severe co-morbidities [11,12]. Bariatric surgeries are classified as either mal-absorptive or restrictive [13] and are effective in reducing obesity-associated co-morbidities such as chronic hypertension and diabetes [13,14] and in improving spontaneous fecundity and pregnancy outcome [15]. However, data regarding the effects of bariatric surgery on the reproductive outcome of infertile women undergoing IVF are still scarce.

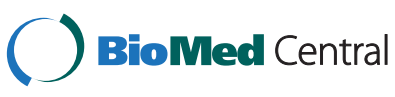

C 2014 Tsur et al.; licensee BioMed Central Ltd. This is an Open Access article distributed under the terms of the Creative Commons Attribution License (http://creativecommons.org/licenses/by/4.0), which permits unrestricted use, distribution, and reproduction in any medium, provided the original work is properly credited. The Creative Commons Public Domain Dedication waiver (http://creativecommons.org/publicdomain/zero/1.0/) applies to the data made available in this article, unless otherwise stated. 
Prompted by the aforementioned observations, we aimed to investigate the effect of bariatric surgery on ovarian stimulation characteristics and IVF treatment cycle outcome in infertile patients undergoing IVF treatment, both prior to and following bariatric surgery.

\section{Methods}

The study population consisted of all female patients admitted to our department between January 2005 and June 2014 with a history of bariatric surgery. We included only patients who underwent IVF treatment cycles both prior to and following bariatric surgery. The study was approved by the institutional IRB of Sheba Medical Center. Stimulation protocols employed for induction of follicular growth were previously described [16] and included long GnRH agonist protocol, flare $\mathrm{GnRH}$-agonist protocol and the multidose $\mathrm{GnRH}$ antagonist protocol. Gonadotropines used included either recombinant FSH or HMG.

Data on patient age, BMI, and variables related to infertility treatment were collected from the files. Ovarian stimulation characteristics, number of oocytes retrieved, embryo quality, and number of embryos transferred during the patients' IVF cycles before and after bariatric surgery were compared. Embryo classification was based on the individual scoring parameters according to pre-established definitions [17]. A top-quality embryo (TQE) was defined as seven or more blastomeres on day 3, equal sized blastomeres, and $<20 \%$ fragmentation; all other embryos were categorized as poor quality.
Statistical analysis was performed using Wilcoxon rank sum test and Chi square, as appropriate $\mathrm{p}<0.05$ was considered significant.

\section{Results}

Out of 9869 patients treated in our IVF unit during the study period, 18 had a history of bariatric surgery. Seven such patients underwent an IVF treatment cycle both before and after bariatric surgery. Five patients underwent sleeve gastrectomy and two gastric banding. Mean patient age \pm SD at IVF cycles before and after the operation were $34.1 \pm 5.69$ years and $36.8 \pm 6.1$ years respectively. Reasons for IVF included male factor infertility $(n=2)$, PCOS $(n=2)$, genetic $(n=1)$, unexplained $(n=1)$ and age $(n=1)$. Mean \pm SD intervals between the operation and the subsequent IVF treatment cycle and between the consecutive IVF cycles were $15.6 \pm 7.9$ months and $28.4 \pm 29.0$ months respectively. All the patients had their weight stabilized before starting the IVF treatment, as the metabolic parameters vary widely during the rapid weight-loss phase after the surgery. Of the seven patients, following the operation two had a normal BMI, two were overweight and three remained obese (of them two remained morbid obese).

The clinical characteristics of patients' IVF cycles before and after the bariatric surgery are shown in Tables 1 and 2. BMI was significantly reduced after the operation $(43.1 \pm 3.3$ vs. $29.6 \pm 7.33, \mathrm{p}=0.018)$. Moreover, in two women severe co-morbidities (diabetes and hypertension) completely resolved following bariatric surgery without any additional treatment.

Table 1 IVF treatment cycle characteristics of each patient undergoing IVF: last cycle before and first cycle after bariatric surgery

\begin{tabular}{|c|c|c|c|c|c|c|c|c|c|c|c|}
\hline patient & $\begin{array}{l}\text { Bariatric } \\
\text { surgery }\end{array}$ & age & BMI & $\begin{array}{l}\text { Total \# of } \\
\text { Gonadotropin } \\
\text { ampoules }\end{array}$ & $\begin{array}{l}\text { Days of } \\
\text { stimulation }\end{array}$ & $\begin{array}{l}\text { Peak E2 } \\
\text { (pmol/L) }\end{array}$ & $\begin{array}{l}\text { \# of } \\
\text { oocytes } \\
\text { retrieved }\end{array}$ & $\begin{array}{l}\text { \# of MII } \\
\text { oocytes }\end{array}$ & $\begin{array}{l}\text { \# of top } \\
\text { quality } \\
\text { embryos }\end{array}$ & $\begin{array}{l}\text { \# of } \\
\text { embryos } \\
\text { transferred }\end{array}$ & $\begin{array}{l}\text { Type of } \\
\text { Bariatric } \\
\text { surgery }\end{array}$ \\
\hline \multirow[t]{2}{*}{$\# 1$} & Before & 38 & 39.3 & 66 & 11 & 3025 & 6 & 3 & 0 & 2 & Sleeve gastrectomy \\
\hline & After & 40 & 29.8 & 66 & 11 & 2562 & 4 & 4 & 0 & 4 & \\
\hline \multirow[t]{2}{*}{ \# 2} & Before & 36 & 42.7 & 50 & 11 & 4302 & 19 & 14 & 6 & 2 & Sleeve gastrectomy \\
\hline & After & 42 & 25.6 & 40 & 11 & 4346 & 9 & 9 & 1 & 3 & \\
\hline \multirow[t]{2}{*}{$\# 3$} & Before & 43 & 42 & 78 & 13 & 4167 & 2 & 2 & 1 & 2 & Sleeve gastrectomy \\
\hline & After & 44 & 37.6 & 66 & 11 & 1065 & 1 & 1 & 0 & 1 & \\
\hline \multirow[t]{2}{*}{ \# 4} & Before & 26 & 47.8 & 71.5 & 25 & 1374 & \multicolumn{4}{|c|}{ Retrieval cancelled - lack of ovarian response } & Gastric banding \\
\hline & After & 27 & 38.9 & 30 & 10 & 4826 & 13 & 8 & 1 & 1 & \\
\hline \multirow[t]{2}{*}{ \# 5} & Before & 32 & 45.8 & 72 & 12 & 1158 & 7 & 5 & 1 & 4 & Sleeve gastrectomy \\
\hline & After & 35 & 33 & 32 & 8 & 5444 & 10 & 8 & 3 & 3 & \\
\hline \multirow[t]{2}{*}{ \# 6} & Before & 29 & 39.1 & * & * & * & 15 & 10 & $*$ & 2 & Gastric banding \\
\hline & After & 31 & 20.7 & $*$ & * & * & 20 & 16 & 3 & 4 & \\
\hline \multirow[t]{2}{*}{ \# 7} & Before & 35 & 45.2 & 78 & 19 & 4271 & 12 & 12 & 0 & 3 & Sleeve gastrectomy \\
\hline & After & 39 & 21.5 & 33 & 13 & 4451.6 & 8 & 8 & 3 & 2 & \\
\hline
\end{tabular}

*Data missing. 
Table 2 Summary of IVF treatment cycle characteristics of all patients undergoing IVF: last cycle before and first cycle after bariatric surgery

\begin{tabular}{|c|c|c|c|c|c|c|c|}
\hline \multicolumn{4}{|l|}{ сусіе arter Darratric surgery } & \multicolumn{4}{|c|}{ yle arter Dariatric surgery (cominiued) } \\
\hline & Before surgery & After surgery & $\mathrm{p}$ & $\%$ of top-quality embryos & & & \\
\hline BMI & & & & Mean \pm SD & $28.2 \% \pm 29.6$ & $37.2 \pm 23.59$ & 0.78 \\
\hline Mean \pm SD & $43.1 \pm 3.3$ & $29.6 \pm 7.3$ & 0.018 & Median & 25.0 & 42.8 & \\
\hline Median & 42.7 & 29.8 & & Range & $0-66$ & $0-75$ & \\
\hline Range & $39.1-47.8$ & $20.7-38.9$ & & Number of embryos & & & \\
\hline Total number of gonadotropin & & & & transferred & & & \\
\hline ampoules used & & & & Mean \pm SD & $2.5 \pm 0.8$ & $2.8 \pm 1.1$ & 0.51 \\
\hline Mean \pm SD & $69.3 \pm 10.5$ & $44.5 \pm 17.0$ & 0.043 & Median & 2 & 3 & \\
\hline Median & 71.7 & 36.5 & & Range & $2-4$ & $1-4$ & \\
\hline Range & $50-78$ & $30-66$ & & Number of embryos frozen & & & \\
\hline Days of stimulation & & & & Mean \pm SD & $1.8 \pm 2.0$ & $0.8 \pm 1.6$ & 0.28 \\
\hline Mean $\pm S D$ & $15.2 \pm 5.6$ & $10.7 \pm 1.6$ & 0.068 & Median & 1.5 & 1.5 & \\
\hline Median & 12.5 & 11 & & Range & $0-4$ & $0-4$ & \\
\hline
\end{tabular}

Peak $E_{2}(p m o l / L)$

Mean \pm SD

Median

Range

$3049.5 \pm 1462.6$

3596

$3782.4 \pm 1642.5 \quad 0.46$

$1158-4302$

4398.8

1065.0-5444.0

Number of follicles $\geq 15 \mathrm{~mm}$ on day of $h C G$ administration

Mean \pm SD

$3.8 \pm 2.3$

$7.0 \pm 4.2$

Median

3

Range

8

$1-11$

Number of oocytes retrieved

Mean \pm SD

$10.1 \pm 6.3$

$8.7 \pm 6.5$

Median

9.5

Range

2-19

9

$1-20$

Number of MII oocytes

Mean \pm SD

\section{$7.7 \pm 5.0$}

$7.7 \pm 5.0$

1.0

Median

Range

7.5

2-14

8

1-16

$\%$ MII of all oocytes retrieved

Mean \pm SD

$79 \% \pm 21.3$

$90.9 \pm 11.5$

0.1

Median

74

Range

Fertilization rate (\%)

Median

Range

50-100

100

$71.7 \% \pm 21.5$

76-100

78.6

$67.9 \pm 26.5 \%$

50

50-100

44-100

Number of top-quality embryos

Mean \pm SD

\section{$1.6 \pm 2.5$}

Median

Range

1

0-6
Table 2 Summary of IVF treatment cycle characteristics of all patients undergoing IVF: last cycle before and first cycle after bariatric surgery (Continued)

$\%$ of top-quality embryos

Following the surgery, patients required significantly fewer gonadotropin ampoules. There were no differences between the cycles in peak estradiol level, number of follicles $\geq 15 \mathrm{~mm}$ on day of hCG administration, the number of oocytes retrieved, MII oocytes, fertilization rates or number of TQE.

One of the patients conceived from embryos cryopreserved in the cycle following the operation. This pregnancy ended in missed abortion in the first trimester. Other patients did not conceive in the first cycle after the surgery.

\section{Discussion}

In this preliminary case series we observed a significant decrease in the total number of gonadotropin ampoules required during IVF cycle following bariatric surgery, with no adverse effects on the number of follicles $\geq 15 \mathrm{~mm}$, number of oocytes retrieved and number of MII oocytes.

To the best of our knowledge, this is the first comparison of IVF cycle characteristics prior to and following bariatric surgery. To date, few reports have described treatment outcomes of infertile female patients undergoing IVF following bariatric surgery. Doblado et al. published their description of five patients who underwent IVF treatment following bariatric surgery [18]. All their patients conceived in the first or second IVF cycle following the operation, and none experienced complications during or after IVF treatment cycles. The authors concluded that bariatric surgery is a safe procedure in women undergoing artificial reproduction techniques. Christofolini et al. compared ovarian stimulation parameters and treatment outcome among three different groups of patients: (a) 29 patients after bariatric surgery [average BMI $26.6 \mathrm{~kg} / \mathrm{m}^{2}$, range (22.8-35.8)]; (b) 57 obese patients [average BMI $32.8 \mathrm{~kg} / \mathrm{m}^{2}$, range (30.146.2)]; and (c) 94 normal-BMI and overweight patients 
[average BMI $23.5 \mathrm{~kg} / \mathrm{m}^{2}$, range (17.5-29.6)] undergoing their first IVF cycle [19]. They reported significantly fewer follicles, oocytes retrieved, and mature oocytes among patients with a prior bariatric surgery as compared with patients from the two other groups. Although the difference was statistically significant, it seems clinically meaningless, since the mean number of follicles and oocytes retrieved were 5 and 5 (respectively) in the bariatric surgery group, 6 and 6 in the obese group, and 7 and 6.5 in the normal BMI/overweight group. However, their conclusions might deserve further consideration, as the difference might be attributable to the variability in ovarian reserve parameters among patients rather than the intervention.

The effect of bariatric surgery on female fertility and ovarian reserve is of specific significance, given the reported increase in the number of bariatric surgeries [20]. Therefore, evaluating the effects of this operation on female fertility has become quite relevant. Radical decreases in BMI after the operation have previously been described. The significant decrease in the number of FSH ampoules required after the surgery is associated both with reduced treatment costs and increased patient comfort, due to fewer injections.

Moreover, while the radical decrease in weight following surgery usually improves metabolic impairments such as diabetes, it might also cause deficiencies in iron, folate, vitamin B12, calcium, and vitamin D [21]. Animal and human data suggest that these nutrients might play a role in folliculogenesis and oocyte maturation [22-24], with deficiencies possibly causing detrimental effects on fertility.

Time to start treatment again was tailored by patient's age, weight reduction and metabolic parameters. Possibly some of the beneficial effects of bariatric surgery on fertility were obscured by the time passed and the potential decrease in ovarian reserve due to aging. Historically women undergoing bariatric surgery were advised to delay pregnancy for 12 to 24 months after surgery. This recommendation was intended to both optimize weight loss and minimize adverse effects of nutritional deficiencies $[25,26]$. Recent studies and guidelines emphasize the importance of nutritional balance rather than the time from surgery to conception [27]. It might make sense to offer this treatment option to younger patients, seeking for infertility treatments, as in the older population the delay in time might interfere with fertility treatment results.

The main limitations of our study are the small sample size (seven patients) and the long period between the operation and the subsequent IVF treatments. In order to demonstrate, for example, a difference of $30 \%$ in the number of oocytes retrieved at a power of $80 \%$ and an alpha value of $5 \%$ using the uncorrected chi-squared test, 33 study subjects would be needed. This is a preliminary study and the sample size was limited by the unique methodology comparing IVF cycle characteristics in the same patients prior to and following bariatric surgery and the relative rarity of such patients.

\section{Conclusions}

These preliminary case series suggest that bariatric surgery for weight reduction seems to be a safe and beneficial treatment option for morbidly obese infertile patients. Further studies are needed to evaluate the long-term effects of bariatric surgery on ovarian reserve or ovarian stimulation characteristics.

\section{Abbreviations}

BMI: Body Mass Index; TQE: Top-Quality Embryo.

\section{Competing interests}

The authors declare that they have no competing interests.

\section{Authors' contributions}

AT- collected data, analyzed it and participated in writing the manuscript. $\mathrm{RO}$ - planned the study design together with RM and edited the manuscript. $\mathrm{JH}$ - Helped in writing the article, AK - Helped in writing the article. RM planned the study design together with $\mathrm{RO}$, analyzed the data, and participated in writing the manuscript. All authors read and approved the final manuscript.

\section{Acknowledgment}

The authors thank Ms. Ilana Gelernter, Department of Statistics and Operations Research, Tel-Aviv University for her statistical analysis.

Received: 16 August 2014 Accepted: 27 November 2014

Published online: 10 December 2014

\section{References}

1. Imes CC, Burke LE: The obesity epidemic: the United States as a cautionary tale for the rest of the world. Curr Epidemiol Rep 2014, 1:82-88.

2. Ogden $\mathrm{CL}$, Carroll MD, Kit BK, Flegal KM: Prevalence of childhood and adult obesity in the United States, 2011-2012. JAMA 2014, 311:806-814.

3. Haslam DW, James WP: Obesity. Lancet 2005, 366:1197-1209.

4. Frøen JF, Arnestad M, Frey K, Vege A, Saugstad OD, Stray-Pedersen B: Risk factors for sudden intrauterine unexplained death: epidemiologic characteristics of singleton cases in Oslo, Norway, 1986-1995. Am J Obstet Gynecol 2001, 184:694-702.

5. Shah DK, Missmer SA, Berry KF, Racowsky C, Ginsburg ES: Effect of obesity on oocyte and embryo quality in women undergoing in vitro fertilization. Obstet Gynecol 2011, 118:63-70.

6. Luke B, Brown MB, Missmer SA, Bukulmez O, Leach R, Stern JE, SART writing group: The effect of increasing obesity on the response to and outcome of assisted reproductive technology: a national study. Fertil Steril 2011, 96:820-825.

7. Kumbak B, Oral E, Bukulmez O: Female obesity and assisted reproductive technologies. Semin Reprod Med 2012, 30:507-516.

8. Orvieto R, Meltcer S, Nahum R, Rabinson J, Anteby EY, Ashkenazi J: The influence of body mass index on in vitro fertilization outcome. Int J Gynaecol Obstet 2009, 104:53-55.

9. Acosta A, Abu Dayyeh BK, Port JD, Camilleri M: Recent advances in clinical practice challenges and opportunities in the management of obesity. Gut 2014, 63:687-695.

10. Tack J, Deloose E: Complications of bariatric surgery: dumping syndrome, reflux and vitamin deficiencies. Best Pract Res Clin Gastroenterol 2014, 28:741-749.

11. Gastrointestinal surgery for severe obesity. NIH consensus development conference, March 25-7, 1991. Nutrition 1996, 12:397-404.

12. Expert panel report: Guidelines (2013) for the management of overweight and obesity in adults. Obesity 2014, 22:S41-S410.

13. DeMaria EJ: Bariatric surgery for morbid obesity. N Engl J Med 2007, 356:2176-2183. 
14. Buchwald $H$, Avidor $Y$, Braunwald E, Jensen MD, Pories W, Fahrbach $\mathrm{K}$, Schoelles K: Bariatric surgery: a systematic review and meta-analysis. JAMA 2004, 292:1724-1737.

15. Tan O, Carr BR: The impact of bariatric surgery on obesity-related infertility and in vitro fertilization outcomes. Semin Reprod Med 2012, 30:517-528.

16. Baum M, Machtinger R, Yerushalmi GM, Maman E, Seidman DS, Dor J, Hourvitz A: Recurrence of empty follicle syndrome with stimulated IVF cycles. Gynecol Endocrinol 2012, 28:293-295.

17. Ziebe S, Lundin K, Janssens R, Helmgaard L, Arce JC, Group MMvRFivFT: Influence of ovarian stimulation with HP-hMG or recombinant FSH on embryo quality parameters in patients undergoing IVF. Hum Reprod 2007, 22:2404-2413.

18. Doblado MA, Lewkowksi BM, Odem RR, Jungheim ES: In vitro fertilization after bariatric surgery. Fertil Steril 2010, 94:2812-2814.

19. Christofolini J, Bianco B, Santos G, Adami F, Christofolini D, Barbosa CP: Bariatric surgery influences the number and quality of oocytes in patients submitted to assisted reproduction techniques. Obesity (Silver Spring) 2014, 22:939-942.

20. Santry HP, Gillen DL, Lauderdale DS: Trends in bariatric surgical procedures. JAMA 2005, 294:1909-1917.

21. Guelinckx I, Devlieger R, Vansant G: Reproductive outcome after bariatric surgery: a critical review. Hum Reprod Update 2009, 15:189-201.

22. Corduk N, Abban G, Yildirim B, Sarioglu-Buke A: The effect of vitamin D on expression of TGF beta1 in ovary. Exp Clin Endocrinol Diabetes 2012, 120:490-493.

23. Inpanbutr N: Association between calbindin-D28K and oogenesis in ovaries of chicken embryos in vitro. Am J Vet Res 1994, 55:1341-1346.

24. Steegers-Theunissen RP, Twigt J, Pestinger V, Sinclair KD: The periconceptional period, reproduction and long-term health of offspring: the importance of one-carbon metabolism. Hum Reprod Update 2013, 19:640-655.

25. Marceau P, Kaufman D, Biron S, Hould FS, Lebel S, Marceau S, Kral JG: Outcome of pregnancies after biliopancreatic diversion. Obes Surg 2004, 14:318-324

26. Beard JH, Bell RL, Duffy AJ: Reproductive considerations and pregnancy after bariatric surgery: current evidence and recommendations. Obes Surg 2008, 18:1023-1027.

27. American College of Obstetricians and Gynecologists: ACOG Committee opinion no. 549: obesity in pregnancy. Obstet Gynecol 2013, 121:213-217.

doi:10.1186/s13048-014-0116-0

Cite this article as: Tsur et al:: Does bariatric surgery improve ovarian stimulation characteristics, oocyte yield, or embryo quality?. Journal of Ovarian Research 2014 7:116.

\section{Submit your next manuscript to BioMed Central and take full advantage of:}

- Convenient online submission

- Thorough peer review

- No space constraints or color figure charges

- Immediate publication on acceptance

- Inclusion in PubMed, CAS, Scopus and Google Scholar

- Research which is freely available for redistribution 